\title{
Téoros
}

Revue de recherche en tourisme

\section{D’une « politique nationale de prestige » à un « marketing}

\section{urbain $»$}

\section{Les formes d'attractivité dans les politiques de valorisation des} trois « Capitales de l'Europe »

\section{Vincent Calay}

Volume 26, numéro 2, été 2007

L’attractivité touristique des territoires

URI : https://id.erudit.org/iderudit/1070942ar

DOI : https://doi.org/10.7202/1070942ar

Aller au sommaire du numéro

Éditeur(s)

Université du Québec à Montréal

ISSN

0712-8657 (imprimé)

1923-2705 (numérique)

Découvrir la revue

Citer cet article

Calay, V. (2007). D’une " politique nationale de prestige » à un « marketing urbain " : les formes d'attractivité dans les politiques de valorisation des trois

« Capitales de l’Europe ». Téoros, 26(2), 19-26. https://doi.org/10.7202/1070942ar 


\section{D'une "politique nationale de prestige " à un " marketing urbain "}

\section{Les formes d'attractivité dans les politiques de valorisation des trois «Capitales de l'Europe »}

\begin{abstract}
Vincent Calay
Depuis le Sommet d'Édimbourg de 1992, Bruxelles, Luxembourg et Strasbourg sont officiellement reconnues comme les trois principaux sièges d'institutions européennes. Cependant, cette reconnaissance de leur statut de siège s'est de plus en plus fréquemment transformée en une désignation comme "Capitale de l'Europe » (Calay, 2003). Dans cet article ${ }^{1}$, je tente de montrer comment l'apparition de cette expression coïncide avec l'émergence de nouveaux imaginaires de la valorisation de l'implantation urbaine de l'Union européenne ${ }^{2}$. En effet, alors que la logique du « siège " fut étroitement liée à la politique de prestige de l'État et au bien commun national, la logique liée à la " capitale » fait - paradoxalement - plus nettement droit à des imaginaires locaux auparavant marginaux ou inexistants, notamment ceux de la ville consommée, de la ville participative ou de la ville culturelle. Cette complexification, liée à l'implication d'un nombre croissant d'acteurs publics et privés dans la problématique de l'implantation européenne dans la ville, s'identifie dans de nombreuses controverses sur la qualité des espaces aménagés pour accueillir l'Union et donc les formes données à leur valorisation.
\end{abstract}

Je m'intéresse ici à trois controverses liées à la présence du Parlement européen dans chacune des trois villes. L'étude de ces controverses permet de souligner la polarisation des débats autour de différentes formes de justifications (Boltanski et Thévenot, 1993) développées par les acteurs. Ainsi s'esquisse au terme de l'article une typologie des différents imaginaires 3 qui structurent l'implantation du Parlement européen. L'implantation bruxelloise se caractérise principalement par une

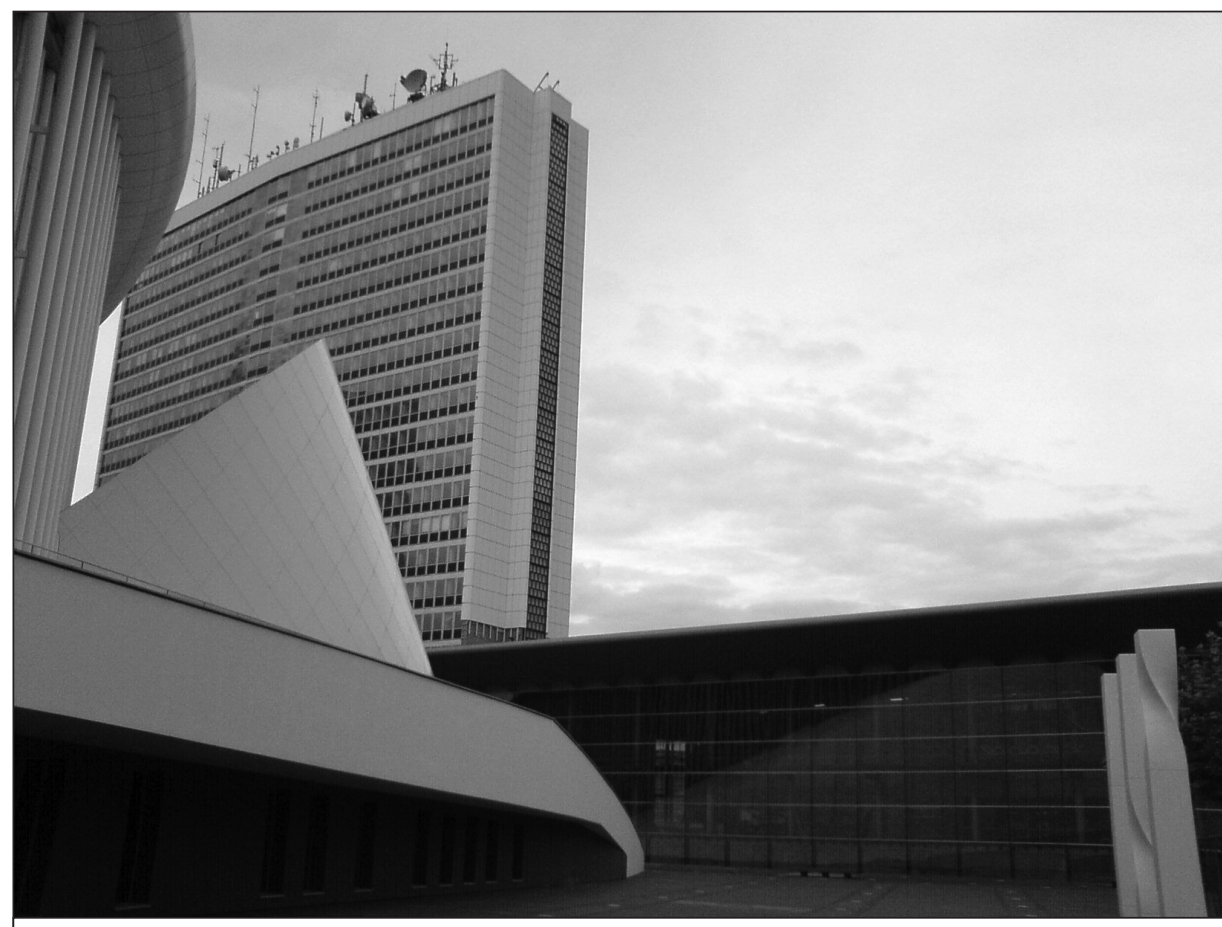

La Hochhaus sur la Place de l'Europe, plus haut et plus ancien gratte-ciel du Grand-Duché de Luxembourg et symbole de l'implantation européenne sur son territoire, est aujourd'hui intégré à un complexe administratif et culturel de première importance pour la ville et le pays.

Photo : Vincent Calay

polarisation des justifications sécuritaires et fonctionnelles, d'une part, et participatives ou culturelles, de l'autre. Par contre, l'implantation luxembourgeoise apparaît plutôt tendue entre une justification liée au bien commun et à la culture, d'une part, et à la ville consommée, de l'autre. Enfin, l'implantation strasbourgeoise souligne les tensions entre des justifications de type sécuritaire et fonctionnel, des justifications liées au bien commun, à la culture ou à la ville consommée. De tels contrastes dans les justifications de la valorisation renvoient à des controverses sur l'usage des espaces du Parlement européen entre différents acteurs, principalement européens et locaux en charge du tourisme ou locaux porte-parole des revendications participatives ou culturelles. En effet, les institutions européennes se préoccupent à titre principal de la qualité ergonomique et sécuritaire de leurs espaces de travail, alors que les autorités touristiques ou les associations spécialisées dans l'usage des espaces publics revendiquent maintenant un droit d'accès à ces espaces européens. Aussi ces espaces se présentent-ils aujourd'hui comme des lieux hybrides où se négocie une transition dans leurs usages et dans leur définition comme éléments intégrés à l'ensemble de l'espace urbain. 
Le développement de ce canevas général de réflexion s'organise en trois parties. Dans une première, je réfléchis à la notion d'attractivité et de valorisation à partir de certains textes produits au sein des études urbaines afin de souligner le relatif consensus développé dans ce champ disciplinaire autour de l'idée de la domination, dans les économies urbaines postfordistes, d'un "compromis de l'attractivité » associant métropolisation et patrimonialisation. Dans une deuxième partie, j'interroge cette idée de "compromis de l'attractivité ", en soulignant les spécificités de son émergence autour de la question de l'implantation européenne dans les trois villes. Enfin, dans une troisième partie, je relativise cette idée par le biais de l'étude de controverses liées à l'implantation du Parlement européen en montrant que la question de l'attractivité de l'espace étudié relève d'un domaine conflictuel où se dessinent des rapports de force entre diverses formes de valorisation des relations entre l'Europe et la ville.

\section{Les nouvelles formes de " compromis de l'attractivité " dans les politiques d'aménagement et de valorisation}

Les études urbaines insistent depuis une quinzaine d'années sur le phénomène de métropolisation qui caractérise les économies urbaines postfordistes (Antier, 2005 ; Hall, 1993 ; Sassen, 2006 ; Vandermotten, 1993). D’après ces écrits, le phénomène génère un renforcement des zones métropolitaines centrales où se développent les fonctions de commandement économique et déclinent les fonctions industrielles. Dans ce contexte, se développe un processus d'esthétisation de l'espace urbain qui inscrit l'attractivité dans de nouveaux instruments d'aménagement: les plans stratégiques de développement (Genard et Bergilez, 2002; Calay et Genard, 2006 ; Evans, 2003 ; Vandermotten, 1993).

Il est généralement admis qu'un tel processus de métropolisation engendre un changement d'échelle dans les politiques. II amène à un recentrage sur la "question locale » (Bourdin, 2000) qui place la régulation des politiques d'aménagement non plus à l'échelle étatique, mais à l'échelle de la ville. Ce changement d'échelle suppose une autonomisation des autorités locales dans les politiques de gestion de la ville.
Ainsi, les capitales nationales où l'emprise de l'État demeure importante présentent des cas d'études de premier intérêt (Taylor et al., 1993 ; Strom, 2001).

Le recentrage à l'échelle locale s'accompagne d'un processus d'esthétisation des politiques d'aménagement qui se manifeste autant dans le phénomène généralisé de «patrimonialisation» (Gravari-Barbas et Guichard-Anguis, 2003) que dans les transformations de la commande publique (Champy, 2001) ou privée (Evans, 2003 ; McNeill, 2005) en architecture. Ce tournant amène à penser que se dessine, à l'échelle métropolitaine, un nouveau compromis, celui de l'attractivité 4 . Un tel compromis redéploie les actions publiques d'aménagement autant sur le plan politique (protection du patrimoine, concours) que technique (managérialisation) et esthétique (rétrospective ou prospective). Les analystes de ce compromis soulignent qu'il s'organise généralement à deux échelles spatiales: celle de l'attractivité locale et celle de l'attractivité internationale (Rosemberg, 2000 ; Ingallina, 2001). S'insèrent dans la première forme d'attractivité, d'une part, la question de la relance de la croissance démographique dans les centres urbains après une période de périurbanisation et, d'autre part, la problématique de la communication politique. Dans la seconde, s'intègrent l'essor du tourisme urbain (Cazes et Potier, 1996) et la question des investissements directs étrangers (Carrière, 2000). Ainsi, dans le contexte de ce compromis postfordiste de l'attractivité des villes, la question patrimoniale a connu des développements liés au réaménagement des quartiers centraux, souvent entamé dans le courant des années 1980 (Linossier et al., 2004). Néanmoins, la conservation du patrimoine bâti et sa valorisation ont elles-mêmes subi quelques transformations. C'est ainsi que, par exemple, plusieurs débats ont émergé à propos de la conservation de l'architecture moderniste des années 1960 à 1970 (Vayssière, 2001).

Dans ce contexte, il apparaît intéressant de s'interroger aujourd'hui sur les relations qu'entretiennent le phénomène de métropolisation et celui de patrimonialisation à l'intérieur de ce nouveau "compromis de l'attractivité ». On peut en effet se questionner sur les dynamiques à l'œuvre de nos jours dans de nombreuses villes européen- nes où à la fois émergent des controverses sur la valorisation du patrimoine bâti des années 1960-1970 et sur la production de bâtiments monumentaux, esthétiquement attractifs et inscrits dans le star-system architectural contemporain.

\section{Du statut de " siège d'institutions européennes " à celui de "Capitale de l'Europe ": les formes de compromis de l'attractivité dans les politiques urbaines bruxelloises, luxembourgeoises et strasbourgeoises}

Les villes sièges d'institutions européennes présentent de ce point de vue des cas d'étude de premier intérêt. En effet, avec l'essor des politiques d'attractivité, on y a vu éclore une nouvelle problématisation de la valorisation de la présence européenne. Ainsi, trois politiques ont tendu à se rejoindre à partir du milieu des années 1980 et se sont rapprochées de façon décisive au début des années 2000: la politique du siège, la politique d'aménagement et la politique de valorisation de la ville.

La politique du siège est fondée sur les obligations supportées par l'État hôte des institutions européennes. Cette politique concerne donc principalement la fourniture des infrastructures d'accueil nécessaires au bon fonctionnement des institutions et relève dès lors d'une coordination entre le ministère des Affaires étrangères, le ministère des Travaux publics et le ministère du Budget. Trois dispositifs particuliers structurent la politique du siège dans chacun des États. Dans les cas belge et luxembourgeois, cette politique a occasionné la mise en place de comités interministériels organisés autour du ministère des Affaires étrangères. Par contre, dans le cas français, la politique du siège relève directement du ministre délégué aux Affaires européennes. Néanmoins, la centralité de l'État dans la politique du siège paraît s'amoindrir aujourd'hui au profit des autorités locales. En effet, s'observe à Bruxelles et à Strasbourg un investissement des institutions locales dans la gestion de la présence européenne. C'est particulièrement le cas à Bruxelles où la création de la fonction de «Madame Europe » en 2005 est étroitement liée à la volonté de la région de réinvestir le quartier européen, jusque-là peu 
intégré aux politiques d'aménagement bruxelloises. Dans le cas strasbourgeois, l'émancipation de la tutelle étatique est plutôt liée à une évolution de la valorisation du quartier européen vers un aménagement dense et à petite échelle des institutions du Conseil de l'Europe et à la liaison du quartier au réseau de tramway. À Luxembourg, la situation est très différente car l'État conserve un monopole presque complet dans l'aménagement du quartier. Le Comité de coordination se charge de négocier les demandes formulées par les institutions européennes en liaison étroite avec le Fonds d'urbanisation et d'aménagement du plateau de Kirchberg qui encadre l'ensemble des aménagements du quartier.

Ainsi, la politique d'aménagement, si elle est isolée par l'analyse, s'avère étroitement liée à la politique du siège. Cependant, son traitement autonome permet de mieux appréhender sa complexité et les effets structurants des débats architecturaux et urbanistiques locaux. Le cas bruxellois se distingue par sa complexité et son éclatement. De multiples acteurs publics et privés interviennent dans la politique d'aménagement du quartier. En effet, le quartier européen bruxellois - dont l'aménagement s'est principalement organisé par le jeu des relations entre les institutions européennes et la promotion immobilière privée - est au centre de la critique développée par les associations et les collectifs soucieux de la qualité de l'environnement urbain et de la liberté de son usage. En comparaison, on observe une centralisation accrue des politiques d'aménagement à Luxembourg et à Strasbourg. Les aménagements du quartier européen luxembourgeois demeurent un domaine réservé de l'État qui justifie un traitement différencié du reste de la ville en raison de son statut de zone "d'édifices et d'installations d'intérêt national ${ }^{5}$ ». Cela se manifeste, notamment, du point de vue de la hauteur des bâtiments où la norme des quatre niveaux hors sol n'est pas respectée, contrairement à la zone bancaire du Kirchberg où cette norme ne souffre pas d'exception. L'esthétique du gratte-ciel apparaît donc étroitement liée aux institutions européennes. À Strasbourg, une nouvelle centralisation s'est développée à l'échelle locale. Ainsi, la politique d'aménagement s'y organise principalement au sein de la direction du développement urbain de la Communauté urbaine de Strasbourg
(CUS) en relation avec l'Agence de développement et d'urbanisme de l'agglomération Strasbourgeoise (ADEUS). Notons, néanmoins, que l'action de ces institutions est étroitement liée au cadre réglementaire du contrat triennal, négocié par la CUS avec l'État central français.

La politique de valorisation a émergé à Luxembourg et à Strasbourg dans le courant des années 1990. Elle s'est tout d'abord manifestée par une politique de grands travaux reliant la question technique de l'infrastructure institutionnelle à la question esthétique d'une architecture européenne. Le glissement vers le postmodernisme a donc amené l'esthétique des bâtiments et des aménagements à s'éloigner du registre fonctionnaliste pour se centrer sur la "symbolique européenne " de certains bâtiments institutionnels et aménagements d'espaces publics. Ce processus s'est, en outre, étayé de signatures prestigieuses qui ont inscrit les aménagements dans le système médiatique de l'architecture contemporaine. Cette politique de prestige de l'État a donc initié une réorientation des politiques d'aménagement des quartiers européens des deux villes. À Strasbourg, cela s'est manifesté dans la construction d'un bâtiment prestigieux pour le Parlement européen par le bureau d'architectes parisiens Architecture Studio. À Luxembourg, les politiques d'aménagement ont intégré la rénovation des deux principaux bâtiments européens, la Cour de Justice (1973) et la Hochhaus (1966), à des développements architecturaux et urbanistiques contemporains de stars de l'architecture : Ricardo Boffill, Yeoh Ming Pei, Dominique Perrault et Christian de Portzamparc. Cela témoigne du souci des autorités luxembourgeoises de préserver des éléments du patrimoine architectural luxembourgeois des années 1960 et 1970, mais également de les mettre en valeur au sein d'espaces architecturaux monumentaux d'esthétique contemporaine. Ces politiques ont esquissé une mise en patrimoine des espaces institutionnels européens intégrant historicité de la présence européenne sur le territoire national et esthétique contemporaine. Une telle politique de valorisation axée sur le prestige international de l'État ne s'est pas développée à Bruxelles où le secteur privé a répondu, par l'entremise des pouvoirs publics, à la demande d'infrastructures des institutions européennes.
Cette situation s'est, cependant, progressivement transformée. En effet, au début des années 2000, les autorités locales des trois villes ont intégré la présence européenne dans la rédaction de leur plan stratégique comme axe directeur de leur politique de valorisation. Ces autorités ont ainsi manifesté leur volonté de lier le quartier européen à la ville, notamment par des infrastructures de transport ferroviaire léger, pour les cas de Luxembourg et de Strasbourg. Par la suite, les acteurs institutionnels de la valorisation touristique ont tenté de concevoir des dispositifs d'appropriation des espaces de travail des institutions européennes. Ce mouvement des acteurs institutionnels poursuit des tentatives antérieures d'appropriation des bâtiments par une série d'acteurs privés. Cela fut particulièrement sensible à Bruxelles où des comités d'habitants ou des collectifs alternatifs défenseurs de l'usage public des espaces européens ont manifesté leur volonté de faire de ces espaces des parties de la ville. Différentes initiatives ont ainsi bourgeonné : de la plus radicale, l'occupation des ailes d'une gare en voie d'être détruite, à la plus consensuelle, une exposition sur le destin européen de Bruxelles organisée à l'initiative d'un comité d'habitants, en collaboration avec la Commission européenne, autour du bâtiment du Berlaymont. À Strasbourg, la volonté d'appropriation du bâtiment du Parlement européen s'est illustrée dans la volonté émise par certains intellectuels de réaliser, dans l'enceinte de l'édifice du Parlement, une Université européenne (Geremek et Vincent, 2006). Le processus d'appropriation se déploie également dans les initiatives de l'office du tourisme de la ville qui œuvre pour obtenir des dates de visites garanties pour les touristes individuels afin de faire du bâtiment un des hauts lieux du tourisme strasbourgeois, au même titre que la cathédrale.

Aussi, la question de l'intégration des espaces européens au patrimoine de la ville apparaît-elle au centre de nombreuses controverses sur l'usage des espaces dévolus aux institutions européennes. Par conséquent, des relations intimes se dégagent entre politiques d'aménagement et de valorisation. Une telle proximité semble refléter, à Bruxelles et à Strasbourg, une autonomisation des institutions et des débats locaux dans la défi- 
nition d'un patrimoine monumental européen où le secteur touristique joue un rôle central. Le cas Luxembourgeois présente, lui, la particularité d'un maintien du monopole étatique qui structure la définition du patrimoine monumental européen de façon très autonome par rapport aux débats locaux et à la sphère touristique.

\section{Les distorsions dans les compromis : controverses sur la valorisation de l'implantation urbaine du Parlement européen à Bruxelles, à Luxembourg et à Strasbourg}

Cette partie de l'article analyse les dispositifs qui dynamisent les interactions entre les acteurs des trois politiques qui encadrent la présence européenne sur le territoire des trois villes d'accueil. Ainsi, j'aborde ici, à une autre échelle, les dynamiques de production de territoires urbains par les divers phénomènes d'appropriation dont font l'objet les espaces institutionnels européens et, en particulier, ceux dévolus au Parlement européen. Ce type d'approche, développée notamment dans la littérature francophone qui conceptualise la notion de paysage (Berque, 1995 ; Conan, 1994 ; Gagnon, 2003 ; Roger, 1997), présente la particularité de ne pas figer a priori un espace au sein d'un usage unique, mais de comprendre les différents dispositifs qui structurent la production de la définition d'une ou de plusieurs qualité(s) de l'espace étudié.

Ces espaces sont donc envisagés comme des objets investis par les exigences planifiées des différents acteurs. $Y$ interagissent ainsi autant la planification technique des espaces, définie par l'administration nationale ou européenne dans son programme pour le bâtiment, que la planification esthétique proposée par l'architecte lauréat ou encore la planification d'une appropriation par différents acteurs institutionnels touristiques, associatifs et/ou alternatifs de l'objet construit/aménagé. L'analyse des trois dispositifs suit donc le canevas commun de la compréhension de certaines controverses qui concernent les espaces du Parlement européen en tant qu'objets intégrés au compromis de l'attractivité déployé dans la ville d'accueil.

\section{Controverse 1 :}

production d'un Musée de I'Europe dans le bâtiment du Parlement européen à Bruxelles

L'édifice du Parlement européen bruxellois comporte cinq parties principales disposées de part et d'autre d'une dalle qui recouvre des voies de chemin de fer. Chacune de ces parties est nommée d'un D suivi d'un chiffre qui suit la chronologie de la construction de chacun d'eux: D1, D2, D3, D4 et D5. Ces cinq bâtiments forment aujourd'hui un vaste complexe de bureaux qui intègre un hémicycle et plusieurs salles de réunion. II répond à la demande produite par le Parlement européen en 1985 de bénéficier d'un hémicycle à Bruxelles. Le gouvernement belge, qui ne souhaitait pas répondre directement à cette demande pour des raisons diplomatiques a, cependant, donné l'affaire en sous-traitance à une entreprise privée créée pour l'occasion et financée par de grandes sociétés bancaires belges. L'édification de ce complexe a fait l'objet de multiples controverses que l'on peut classer en trois séries parallèles à l'évolution du chantier. Une première série est liée à la destruction de la brasserie, de maisons d'habitations, de la gare et à la mobilisation corollaire des habitants. Une deuxième série est liée à l'usage de la dalle de couverture du chemin de fer comme espace public. Une troisième est liée aux contraintes de mixité imposées aux deux derniers bâtiments: D4 et D5. Je me concentre dans ce texte sur une controverse de la troisième série qui concerne l'implantation d'un «Musée de l'Europe » dans le D4.P8

Le 6 mars 2003, un accord est intervenu entre les parties concernées par la construction des bâtiments D4 et D5 sur l'aménagement d'un espace socioculturel doté d'une cafétéria publique. Cet accord répond à l'obligation faite au promoteur immobilier de construire en compensation des bureaux une certaine surface de logements, de commerces ou d'équipements collectifs afin de conserver une mixité fonctionnelle dans le quartier. Ce mécanisme s'appelle «charges d'urbanisme ». Ce projet de centre socioculturel s'inscrit également dans le prolongement d'un accord plus ancien sur le maintien du caractère public de la dalle située entre le D3 et les D4 et D5 que le projet de cafétéria est censé stimuler: son ouverture prolongée indépendante des horaires du centre socioculturel est prétendue garante d'une animation du site.

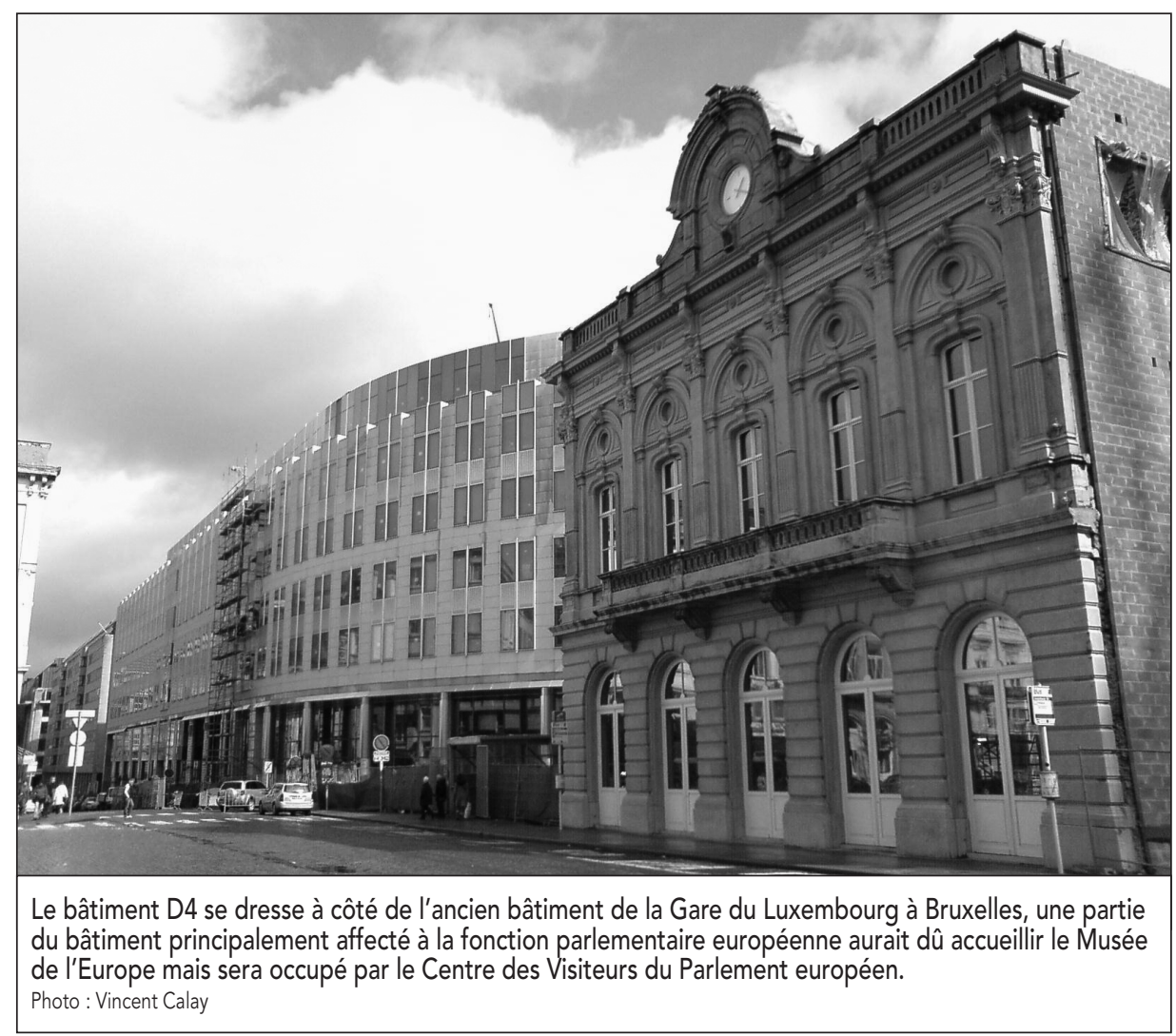


Parallèlement à ces accords de principe, inscrits dans les différentes conventions qui organisent l'histoire juridique des édifices, il existe également une histoire sociale de leur construction, dont les sources sont plus difficilement accessibles. Je poserai donc l'hypothèse que ce centre socioculturel répond à deux types de pressions sur les parties au contrat: I'une liée à des cercles plutôt écologistes défenseurs du maintien du caractère public d'espaces monumentaux qui transforment fondamentalement les structures morphologiques et fonctionnelles du quartier ; l'autre liée à un cercle d'élites soucieuses de valoriser l'implantation européenne à Bruxelles sous un angle culturel étroitement associé au développement du tourisme urbain. Ce deuxième type de pression est issu du développement du projet de "Musée de l'Europe » par une élite europhile bruxelloise bien insérée dans les réseaux de sociabilité belgo-européens (Charléty, 2006). Ce projet privé a d'ailleurs reçu l'aval de différentes autorités publiques belges et européennes. Toutefois, l'ouverture initiale du Parlement européen à l'initiative bruxelloise s'est transformée, début 2006, en une absorption de l'idée de musée dans sa propre politique de communication. Ainsi, le Parlement a rejeté le monopole octroyé tacitement au projet de Musée de l'Europe pour développer son propre "centre des visiteurs ». Ce centre apparait comme une maigre contrepartie au rejet du Musée de l'Europe, puisque les ambitions intellectuelles portées par l'initiative locale - déployer une vision post-nationale du système politique européen - semblent s'être réduites à une histoire assez classique de l'Europe combinée à une présentation traditionnelle de l'institution. Un tel retour de manivelle peut se voir comme une affirmation par l'institution parlementaire d'une régulation serrée de l'accès à ses bâtiments. En effet, partant des contraintes sécuritaires, l'institution se préoccupe principalement de la gestion des circulations sur son site et de la séparation scrupuleuse des visiteurs et des parlementaires. La création d'un centre des visiteurs doté d'une entrée spécifique éloignée de l'entrée principale du Parlement se présente ainsi comme un dispositif sécurisé qui répond aux pressions exercées sur l'institution parlementaire autant par les représentants des usages publics de l'espace que ceux de leur usage éducatif et touristique.

\section{Controverse 2 : \\ production d'un Monument européen dans le bâtiment du Parlement européen à Strasbourg}

Au contraire de Bruxelles, on a opté ab limine dans le bâtiment de Strasbourg la tradition française du Grand Prix de Rome (Champy, 2001) et des Grands Travaux présidentiels (Chaslin, 1985). Les architectes du bâtiment ont donc élaboré un projet architectural qui, au-delà du programme technique requis par le Parlement, intègre un questionnement sur la représentation architecturale de la démocratie européenne. Une telle conception en ferait un monument européen (Debray, 1999) qui questionne les catégories de l'entendement sur la notion. En effet, comme les villes monumentales telles que Washington DC, Brasilia ou Canberra, il apparaît à son échelle comme la projection dans l'espace d'un projet politique qui n'est pas arrêté en substance. Par ailleurs, cette production architecturale d'une représentation de la démocratie européenne s'inscrit dans la volonté de prestige national français intégré à la pour une élaboration architecturale dans

politique du siège. Là s'arrête toutefois le pouvoir de valorisation des autorités étatiques puisque l'institution parlementaire, devenue propriétaire de l'ouvrage, réglemente sévèrement l'accès au bâtiment. L'institution y a ainsi fait intégrer des dispositifs de séparation des circulations entre visiteurs et parlementaires tels que: serrure dans les ascenseurs empêchant de descendre à l'étage des visites; division des circulations dans le bâtiment en trois flux (parlementaires, journalistes et visiteurs) au moyen de portillons escamotables commandés à distance; visites de groupe encadrées, sur réservation et après contrôle d'identité et enregistrement dans les dossiers de l'institution; badges provisoires identifiant les visiteurs.

L'essor d'une politique d'attractivité à l'échelle locale basée sur la présence européenne a mené à une appropriation de plus en plus nette d'un espace aussi prestigieux. Aussi, le secteur touristique local a-t-il intégré le bâtiment du Parlement européen à la représentation de la ville de Strasbourg. À tel point qu'il en est venu à supplanter le bâtiment du Conseil de l'Europe (Calay, 2003). Cette

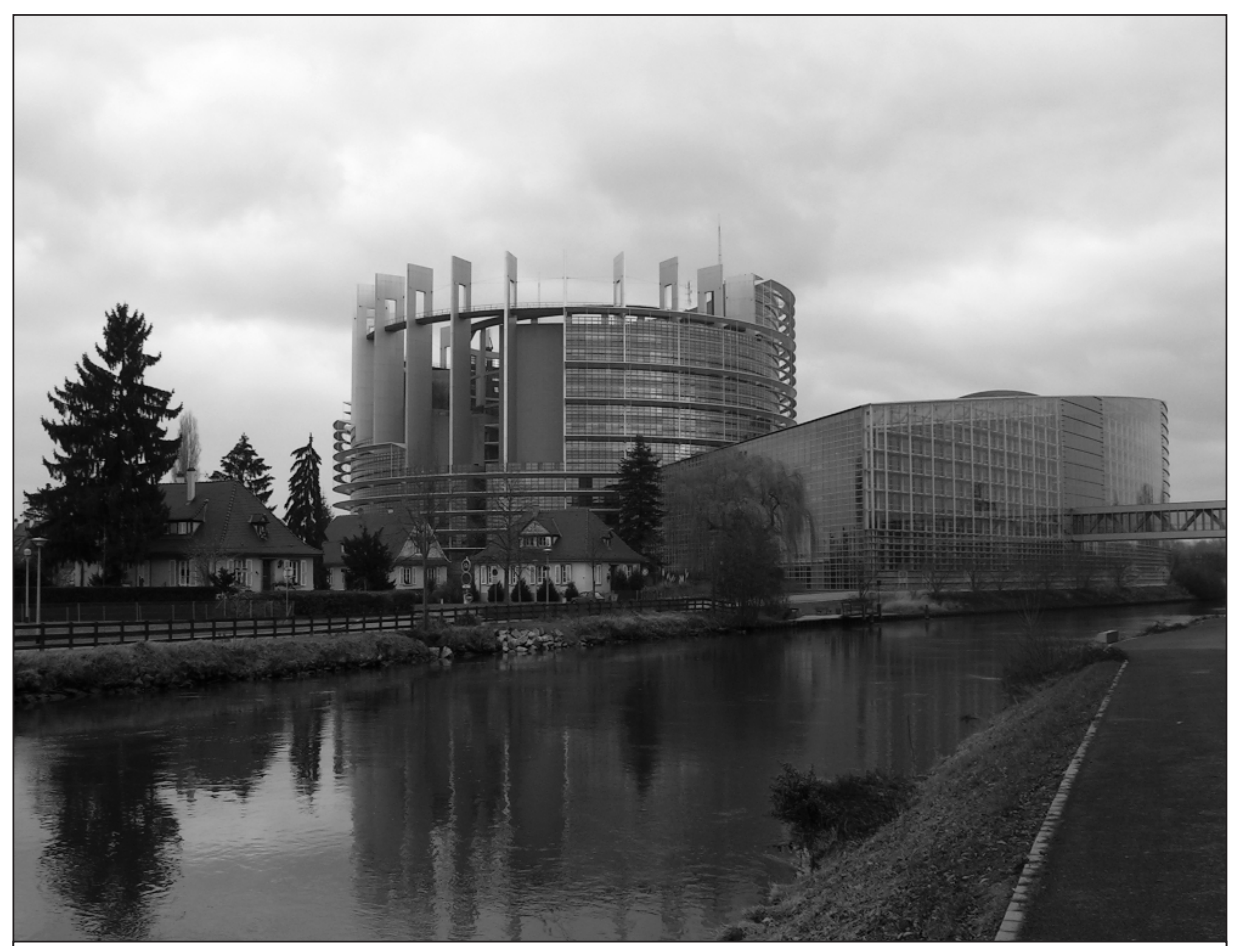

Le Parlement européen de Strasbourg dessiné par Architecture Studio fait aujourd'hui l'objet d'une controverse sur son accessibilité. En effet, malgré son intégration progressive à l'image de la ville, son accès demeure réglementé par la politique de visite de l'institution parlementaire sur laquelle les autorités touristiques locales ont peu d'emprise.

Photo : Vincent Calay 
appropriation par le secteur touristique a poussé à une affirmation progressive de la place du quartier européen dans les itinéraires de visite proposés. Ainsi, par exemple, les bateaux-mouches, attraction principale de la ville, terminent leur visite dans le bassin de l'Ill autour duquel sont implantés le Parlement européen, le Conseil de l'Europe et la Cour européenne des Droits de l'Homme. De même, le plan fourni au visiteur par l'office du tourisme intègre le quartier européen et les édifices. Cette organisation de l'espace par le secteur touristique correspond à l'axe européen qui structure le plan stratégique strasbourgeois.
Cette appropriation progressive du quartier européen et du Parlement européen comme partie du patrimoine monumental de la ville de Strasbourg comporte une controverse principale: la volonté de l'office du tourisme d'obtenir un calendrier de visites garanti pour les visiteurs individuels. En effet, actuellement, le touriste individuel n'a pas accès au bâtiment alors que l'office du tourisme estime que la demande est importante. L'office se voit donc contraint de renvoyer les visiteurs au service des visites du Parlement européen. Cependant, la visite n'est accessible qu'aux groupes préconstitués accrédités auprès de l'institution parlementaire, soit par le service des visites, soit par parrainage ou invitation d'un député. La ten- tative de l'office du tourisme consiste ainsi en une tentative d'imposition des normes propres au monde touristique à l'espace de travail d'une institution dotée de normes sécuritaires. C'est cette confrontation des pouvoirs de régulation de l'accessibilité des bâtiments qui dynamise un processus de patrimonialisation du Parlement européen. Cette controverse sur l'accessibilité prolonge la politique de représentation de Strasbourg comme "Capitale de l'Europe". En effet, l'enjeu pour la ville consiste en une mise à niveau de l'accessibilité du Parlement européen sur la norme courante pour les autres éléments du patrimoine historique de la ville. Cette controverse est donc investie de grandeurs telles que l'usage d'un espace

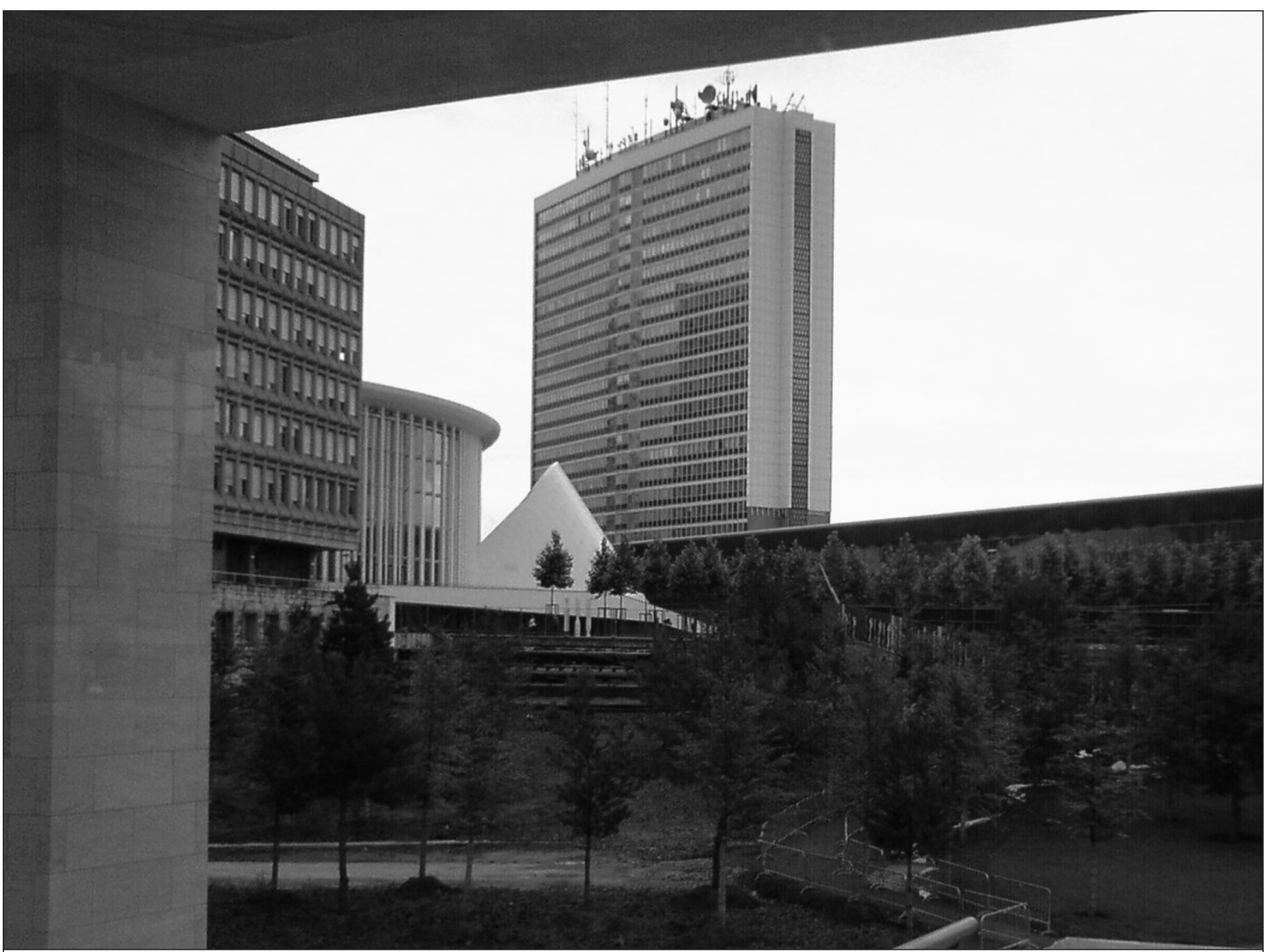

La Place de l'Europe sur le Plateau de Kirchberg à Luxembourg est aujourd'hui en plein réaménagement. Y cohabitent progressivement des infrastructures européennes de Congrès et des infrastructures culturelles développées par des architectes de premier plan comme Christian de Portzamparc ou Yeoh Ming Pei.

Photo : Vincent Calay 
de travail, sa prédéfinition comme objet architectural et la politique d'attractivité menée à l'échelle de la ville.

\section{Controverse 3 : production d'un pôle culturel luxembourgeois dans les espaces institutionnels européens}

Le Parlement européen dispose de son secrétariat à Luxembourg. L'implantation de l'institution dans cette ville ne relève donc pas d'une logique semblable à celle de Bruxelles et de Strasbourg, le Parlement européen n'y tenant pas de séance plénière. II ne faudra donc pas chercher une symbolique particulière de la démocratie représentative européenne telle qu'elle s'est affirmée à Strasbourg ou que l'on tente de développer à Bruxelles.

Ce défaut de bâtiment parlementaire symbolique n'a toutefois pas empêché qu'une problématique semblable à celle analysée dans les deux controverses précédentes se développe. En effet, l'administration parlementaire européenne qui était initialement installée dans trois bâtiments différents sur le plateau de Kirchberg centralise actuellement ses services dans un seul des trois: le bâtiment Konrad-Adenauer dont les travaux d'extension sont sur le point de débuter. Ainsi, actuellement, seul un des trois bâtiments a été libéré par l'institution: la Hochhaus. Le deuxième ne le sera qu'une fois l'extension du Konrad-Adenauer achevée. Une telle politique de regroupement s'opère en étroite collaboration avec le gouvernement luxembourgeois. En effet, celui-ci projette, depuis le début des années 1990, un réaménagement complet du quartier européen, qui concrétise le projet développé par le Fonds d'urbanisation et d'aménagement du plateau de Kirchberg dans le courant des années 1980: faire du plateau une partie de la ville dense et mixte, non une zone purement administrative. Ce cadre général de la mixité a donc bénéficié des initiatives lancées par le Grand-Duché à l'occasion de sa candidature au titre de Capitale européenne de la culture en 2007. C'est ainsi que plusieurs projets d'infrastructures culturelles ont vu le jour autour d'une «Place de l'Europe » dessinée au pied et dans l'axe de la Hochhaus. L'État luxembourgeois a, de la sorte, intégré au quartier des institu- tions européennes des infrastructures culturelles nationales dotées d'une architecture plébiscitée dans le champ médiatique international.

Une telle politique correspond à la volonté du gouvernement luxembourgeois de casser l'image austère de centre financier international développée à partir des années 1960-1970 et de déployer une offre culturelle attrayante pour les élites européennes vivant au Grand-Duché.

La production d'une telle offre esthétique et culturelle par l'État se fait parallèlement à une reconfiguration des politiques menées à l'échelle de la ville de Luxembourg. La mise au point d'un plan stratégique qui inclut la présence européenne comme outil d'attractivité a mené les autorités touristiques de la ville à organiser une offre liée à l'innovation architecturale présente au Kirchberg. Pour y parvenir, un partenariat a dû être mis en place entre l'office du tourisme de Luxembourg et le Fonds Kirchberg pour la production d'une brochure de valorisation. Malgré cela, une controverse est née autour de la problématique de l'accès piétonnier et en autocar aux infrastructures. Ainsi, les visions du fonds et de l'office divergent. L'un se préoccupe des dimensions principalement esthétiques, tandis que l'autre critique le manque de places de stationnement et la difficulté d'accès au plateau depuis le centre historique. Une telle confrontation des expertises sur l'aménagement souligne une forte étanchéité entre les problématiques liées à la politique de prestige et celles associées à la politique de valorisation.

\section{Conclusion}

L'idée, tirée de la littérature produite au sein des études urbaines, de domination d'un " compromis de l'attractivité » dans les économies urbaines post-fordistes mérite, au regard d'études de cas pratiques, une certaine relativisation. En effet, si l'on peut observer une tendance à l'hégémonie de l'idée d'attractivité dans les politiques d'aménagement et de valorisation de l'implantation européenne à Bruxelles, à Luxembourg et à Strasbourg, l'article a montré l'importance des controverses en ce domaine. Controverses qui identifient une zone conflictuelle autour de l'usage des espaces européens et, à ce titre, autour de la valorisation des rapports entre l'Europe et la ville. Ainsi a-t-on pu voir que les objets architecturaux et urbanistiques développés pour accueillir l'institution parlementaire sur le sol des trois villes sont investis de diverses formes d'imaginaires. Ces imaginaires s'organisent selon certains rapports de force qui orientent les dispositifs de valorisation de la relation entre l'Europe et la ville dans l'une ou l'autre direction. En outre, a-t-on vu que l'implantation bruxelloise se caractérise principalement par une polarisation des justifications sécuritaires et fonctionnelles, d'une part, et participatives ou culturelles, de l'autre. Cette polarisation s'est notamment manifestée dans l'échec de l'implantation d'un "Musée de l'Europe » soutenant une certaine définition de l'Europe dans l'enceinte même du Parlement. L'idée fut toutefois réappropriée par l'institution parlementaire qui développe à présent un «centre des visiteurs ». Par contre, l'implantation luxembourgeoise est plutôt apparue tendue entre une justification liée au bien commun et à la culture, d'une part, et à la ville consommée, de l'autre. Le développement de la Place de l'Europe au cœur du quartier européen de Luxembourg montre donc la volonté des autorités nationales de déployer un pôle culturel de prestige, vitrine de l'État luxembourgeois à l'heure de l'essor du capitalisme culturel mondial. Une telle ambition entre en porte-à-faux face aux justifications managériales développées par les porte-parole de la ville de Luxembourg qui se soucient plutôt des dispositifs physiques permettant la consommation de ces infrastructures. Enfin, l'implantation strasbourgeoise souligne les tensions entre des justifications de type sécuritaire et fonctionnel, des justifications liées au bien commun, à la culture ou à la ville consommée. Cela s'illustre principalement dans la controverse associée à l'accessibilité du bâtiment parlementaire strasbourgeois. En conséquence, les justifications sécuritaires de l'institution parlementaire imposent une procédure relativement lourde pour accéder au bâtiment. Cette situation contrevient à la volonté des autorités touristiques de faire du Parlement européen un élément de visite de la ville accessible, au même titre que les autres monuments de la ville, au touriste individuel. Deux conceptions de la ville entrent en conflit dans cette controverse qui poursuit une controverse technique associée aux dispositifs de séparation des circulations au sein de l'édifice parlementaire, renforcés par l'institution pour des raisons sécuritaires car peu développés par les architectes concepteurs de l'édifice. 
Vincent Calay est chercheur à l'Institut de gestion de l'environnement et d'aménagement du territoire (IGEAT) et membre du Groupe de recherche sur l'action publique (GRAP) de I'Université Libre de Bruxelles (ULB). Pour ses recherches, il bénéficie du soutien du Fonds d'encouragement à la recherche de I'ULB

\section{Notes}

1 Cet article est extrait de mes recherches doctorales en sciences politiques et sociales en cours à l'Université Libre de Bruxelles sous le titre provisoire: Aménager et valoriser l'implantation urbaine de l'Union européenne : étude comparée des dispositifs déployés à Bruxelles, à Luxembourg et à Strasbourg. Le propos de cet article a, par ailleurs, fait l'objet d'une conférence à I'Université européenne d'été du Val de Loire le 29 septembre 2006 à Angers, France.

2 Les différents arguments produits sont fondés sur une enquête construite à partir d'entretiens rétrospectifs avec les porte-parole d'acteursclés, d'observations participantes et d'une étude documentaire sur des sources variées: documents officiels (rapports, contrats...), articles de presses, ouvrages spécialisés...

3 Pour davantage de développement sur la notion et son intérêt dans l'étude du tourisme, voir : Calay et Genard (à paraître).

4 Wagenaar (1993) propose une analyse du compromis de la capitale baroque dans les aménagements haussmanniens de Paris. Pour lui, ce compromis se définit dans la production d'une ville fondée sur une rationalisation : la maîtrise intégrale et intégrée des voies de communication terrestre au sein de la ville et de celle d'acheminement / rejet des matières premières nécessaires comme l'eau. Cette approche technique s'associe à une visée politique et esthétique: détruire les taudis jugés insalubres, fournir de nouveaux logements esthétiquement attractifs, produire une ville tout entière monumentale afin de garantir un prestige international au pays. Je propose dans le présent article de penser le compromis de l'attractivité comme une reformulation du cadre qui régule les politiques d'urbanisme. Voir également Lacaze (1997) qui présente une synthèse des divers types de compromis qui jalonnent les politiques d'aménagement urbain.

5 Terminologie du plan général d'aménagement de la Ville de Luxembourg.

\section{Bibliographie}

Antier, Gilles (2005), Les Stratégies dans les grandes métropoles. Enjeux, pouvoirs et aménagements, Paris, Armand Colin.

Berque, Augustin (1995), Les Raisons du paysage. De la Chine antique aux environnements de synthèse, Paris, Hazan.

Boltanski, Luc, et Laurent Thévenot (1993), De la justification. Les Économies de la grandeur, Paris, Gallimard.

Bourdin, Alain (2000), La Question locale, Paris, Presses universitaires de France.

Calay, Vincent (2003), « La Production imaginaire d'une Capitale de l'Europe: vers la territorialisation d'une identité collective européenne ? ", BELGEO, vol. 4, p. 363-384.

Calay, Vincent, et Jean-Louis Genard (2006), «Producing and Understanding Architecture in the Postfordist Times. The Case of Brussels as the Capital of Europe ", dans Hana Gottesdiener et Jean-Christophe Vilatte (dir.), Culture and Communication, Avignon, Proceedings of the XIX ${ }^{\text {th }}$ Congress of the International Association of Empirical Aesthetics, p. 811-815.

Calay, Vincent, et Jean-Louis Genard (dir.) (à paraître), Tourisme et imaginaires urbains, Paris, L'Harmattan, collection «Structures et pouvoirs des imaginaires ».

Carrière, Jean-Paul (2000), « Mondialisation, mobilité internationale et recomposition territoriale ", dans Thierry Paquot et al. (dir.), La Ville et l'Urbain, l'état des savoirs, Paris, La Découverte, p. 146-155.

Cazes, Georges, et Françoise Potier (1996), Le tourisme urbain, Paris, Gallimard.

Champy, Florent (2001), Sociologie de l'architecture, Paris, La Découverte.

Charléty, Véronique (2006), «Bruxelles : Capitale européenne de la culture? L'invention du Musée de l'Europe ", Études européennes, $n^{\circ} 9$, mars.

Chaslin, François (1985), Les Paris de François Mitterrand, Paris, Gallimard.

Conan, Michel (1994), " L'invention des identités perdues ", dans Augustin Berque, Cinq propositions pour une théorie du paysage, Seyssel, Champ Vallon, p. 31-50.

Debray, Régis (1999), « Le monument ou la transmission comme tragédie ", dans Régis Debray (dir.), L'Abus monumental ?, Paris, Fayard.

Evans, Graeme (2003), "Hard-branding the Cultural City - From Prado to Prada", International Journal of Urban and Regional Research, vol. 27, n², p. 417-440.

Gagnon, Serge (2003), L'Échiquier touristique québécois, Québec, Presses de l'Université du Québec.

Genard, Jean-Louis, et Jean-Didier Bergilez (2002), « L'évolution de l'architecture à l'ère de l'esthétisation de la vie quotidienne", Recherches en communication, $\mathrm{n}^{\circ} 18$, p. 133-154.

Geremek, B., et J.-D. Vincent (2006), "Pour une Université de l'Europe", Le Monde, 19 janvier, p. 19.

Gravari-Barbas, Maria, et Sylvie Guichard-Anguis (dir.) (2003), Regards croisés sur le patrimoine dans le monde à l'aube $d u X X I^{e}$ siècle, Presses de l'Université de Paris-Sorbonne.

Hall, Peter (1993), «The Changing Role of Capital Cities: Six Types of Capital City ", dans John Taylor et al. (dir.), Capital Cities - Les Capitales, perspectives internationales International Perspectives, Ottawa, Carleton University Press, p. 69-84.

Ingallina, Patrizia (2001), Le projet urbain, Paris, Presses universitaires de France.

Lacaze, Jean-Paul (1997), Les Méthodes de l'urbanisme, Paris, Presses universitaires de France.

Linossier Rachel et al. (2004), «Entre conflits et synergies. Renouvellement urbain et patrimonialisation ", DISP, vol. 40, n 4, p. 4-12.

McNeill, Donald (2005), « In Search of the Global Architect: the Case of Norman Foster (and Partners) ", International Journal of Urban and Regional Research, vol. 29, n³, p. 501-515.

Roger, Alain (1997), Court traité du paysage, Paris, Gallimard.

Rosemberg, Muriel (2000), Le marketing urbain en question: production d'espace et de discours dans quatre projets de villes, Paris, Anthropos.

Sassen, Saskia (2006), Territory, Authority, Rights: from Medieval to Global Assemblages, Princeton, Princeton University Press.

Strom, Elizabeth (2001), Building the New Berlin. The Politics of Development in Germany's Capital City, Lanham, Lexington Books.

Taylor, John et al. (dir.) (1993), Capital Cities - Les Capitales, perspectives internationales International Perspectives, Ottawa, Carleton University Press.

Vandermotten, Christian (dir.) (1993), Planification et stratégies de développement dans les Capitales européennes, Bruxelles, Éditions de l'Université de Bruxelles.

Vayssière, Bruno (2001), "Patrimonialiser les grands ensembles ? ", dans François Loyer (dir.), Villes d'hier, villes d'aujourd'hui en Europe, Paris, Fayard, p. 365-377.

Wagenaar, Michiel (1993), « Monumental Centre, Picturesque Environs: Contrasting Townscapes and Divergent Land Use in Six European Capitals, 1850-1914 ", dans Christian Vandermotten (dir.), Planification et stratégies de développement dans les Capitales européennes, Bruxelles, Éditions de l'Université de Bruxelles, p 29-47. 